\title{
1 Epidemiological dynamics of viral infection in a marine picoeukaryote
}

2 Luisa Listmann ${ }^{1,2}$, Sarah Heath $^{3}$, Pedro F. Vale ${ }^{3}$, C. Elisa Schaum ${ }^{1,2}$, Sinead Collins ${ }^{3}$

3 1. University of Hamburg, Institute for Marine Ecosystem and Fisheries Science, 22767 Hamburg

4 2. Centre for Earth System Science and Sustainability, 20146 Hamburg

5 3. Institute of Evolutionary Biology, School of Biological Sciences, The University of Edinburgh,

6 Ashworth Laboratories, Edinburgh EH9 3FL, UK

7 Corresponding author: Luisa Listmann, luisa.listmann@uni-hamburg.de

8

9 Key Words: Host-virus dynamics, Ostreococcus, cost of resistance, culturing conditions

\section{Abstract (250 words)}

Ostreococcus tauri is a ubiquitous marine pico-eukaryote that is susceptible to lysis upon infection by its species specific Ostreococcus tauri viruses (OtVs). In natural populations of $O$. tauri, costs of resistance are usually invoked to explain the persistence or reappearance of susceptible individuals in resistant populations. Given the low costs of resistance measured in laboratory experiments with the $O$. tauri/OtV system to date, the question remains of why susceptible individuals persist in the wild at all. Epidemiological models of host and pathogen population dynamics are one useful approach to understand the conditions that can allow the coexistence of susceptible and resistant hosts. We used a SIR (Susceptible-Infected-Resistant) model to investigate epidemiological dynamics under different laboratory culturing regimes that are commonly used in the O.tauri/OtV system. When taking into account serial transfer (i.e. batchcycle lengths) and dilution rates as well as different resistance costs, our model predicts that no susceptible cells should be detected under any of the simulated conditions - this is consistent with laboratory findings. We thus considered an alternative model that is not used in laboratory experiments, but which incorporates one key process in natural populations: host populations are periodically reseeded with new infective viruses. In this model, susceptible individuals re-occurred in the population, despite low costs of resistance. This suggests that periodic attack by new viruses, rather than (or in addition to) costs of resistance, may explain the high proportion of susceptible hosts in natural populations, and underlie the discrepancy between laboratory studies and observations of fresh isolates. 
36 Importance (150 words)

37 In natural samples of Ostreococcus sp. and its associated viruses, susceptible hosts are 38 common. However, in laboratory experiments, fully resistant host populations readily and 39 irreversibly evolve. Laboratory experiments are powerful methods for studying process 40 because they offer a stripped-down simplification of a complex system, but this 41 simplification may be an oversimplification for some questions. For example, laboratory and 42 field systems of marine microbes and their viruses differ in population sizes and dynamics, 43 mixing or migration rates, and species diversity, all of which can dramatically alter process 44 outcomes. We demonstrate the utility of using epidemiological models to explore 45 experimental design and to understand mechanisms underlying host-virus population 46 dynamics. We highlight that such models can be used to form strong, testable hypotheses 47 about which key elements of natural systems need to be included in laboratory systems to 48 make them simplified, rather than oversimplified, versions of the processes we use them to 49 study. 


\section{Introduction}

Surveys of biological diversity over the past decades have shown that viruses infecting marine phytoplankton are one of the most numerically important biological entities on the planet (Suttle 2005). Given their high abundance, and in light of several case studies of the possible roles of viruses in structuring marine microbial populations (Schroeder et al. 2003; Suttle 2007), it is well-accepted that marine viruses play a major top-down role in shaping microbial populations in aquatic systems (Suttle 2007; Brussaard 2004; Weitz and Wilhelm 2012), which can then affect aquatic food webs and nutrient cycles. For example, phytoplankton, which include eukaryotic marine primary producer, play an important role in the biological carbon pump when they are grazed or sink as debris to the ocean floor. Here, infections of phytoplankton populations by marine viruses have the potential to alter dynamics of carbon sequestration (Suttle 2007). To understand how host-virus interactions affect host population dynamics and diversity, prokaryotic host-virus laboratory systems have been developed and produced multiple examples of how viruses can drive evolution in their bacterial hosts at the genome, population and community levels (Koskella and Brockhurst 2014; Westra et al. 2019). However, fewer studies exist for eukaryotic microbes such as phytoplankton and their viruses (Short 2012), limiting our ability to integrate host-virus interactions into our understanding of how marine phytoplankton populations are shaped.

We focus on the marine pico-eukaryote Ostreococcus tauri. Photosynthetic picoeukaryotes are less than $3 \mu \mathrm{m}$ in diameter, and although they are not numerically dominant in oceans, they are important primary producers (Worden, Nolan, and Palenik 2004). O. tauri is globally distributed, and is the smallest free-living eukaryote described to date (Courties et al. 1994). Due to its simple cell structure, available genome sequence and ease of culturing, $O$. tauri and its viruses (Ostreococcus tauri virus; OtV) have been widely used over the past decade as a model organism for studying marine virus infection (E. Derelle et al. 2006; Clerissi, Desdevises, and Grimsley 2012; Clerissi, Grimsley, Ogata, et al. 2014). To date, $O$. tauri has been found via metagenomic analysis in or isolated from several oceanic areas including the Mediterranean, the Atlantic, the Baltic Sea (Clerissi, Grimsley, Subirana, et al. 2014; Šulčius and Holmfeldt 2016; Zeigler Allen et al. 2017, Listmann et al unpubl data). Where OtVs have been isolated and infections investigated, studies found OtVs to be mainly species specific and readily able to infect hosts (Clerissi, Desdevises, and Grimsley 2012). This indicates that in natural populations susceptible cells (S) are probably present at high 
83

84

frequencies whereas resistant $(\mathrm{R})$ lineages are present at low frequencies, which implies that host resistance is either costly, reversible, or both.

In laboratory studies, resistant $(\mathrm{R})$ cells quickly arise from susceptible (S) populations at a rate of at least one in 1000 (Yau et al. 2016). In addition, viral resistance is both cheap and irreversible once it evolves in laboratory culture, even in the subsequent absence of viruses (Heath et al. 2017; Thomas et al. 2011), which suggests that resistance should be widespread in natural populations. Evidence that both genetic and epigenetic changes are involved in resistance (Yau et al. 2016) could explain why a shift from resistance to susceptibility has not been observed in laboratory populations, in that a genetic constraint could prevent reversion or make it extremely rare. However, if resistance is genetically constrained with little or no cost, this begs the question of why susceptible cells are detected in fresh isolates of $O$. tauri (Clerissi, Desdevises, and Grimsley 2012; Evelyne Derelle et al. 2008) and how $\mathrm{S}$ and $\mathrm{R} O$. tauri cells coexist in their natural environment.

A possible way to reconcile these data is to consider that the cost of resistance measured in laboratory culture does not reflect the magnitude of that cost in the ocean, but testing this hypothesis experimentally is challenging on a number of levels. A second nonexclusive explanation is that laboratory rearing conditions, which differ drastically from natural conditions, affect host-virus dynamics in ways that could explain this discrepancy.

One useful way to explore how viruses can interact with, and thus affect the biology of their host populations, is through epidemiological models (Heesterbeek et al. 2015; Grassly and Fraser 2008; Lenski 1988; Gandon and Vale 2014). This approach is rarely used in marine (or even phytoplankton) host-virus systems (Middelboe 2000), and here we show that such models can be useful for understanding both the population dynamics of host populations and the relative contributions of resistant (R) and susceptible (S) cells, as well as the population dynamics of the viral strain to which resistance has evolved. The advantage of using models to understand the dynamics of host-virus systems are two-fold: First, they are an important complement to laboratory studies and surveys of natural populations, especially in marine systems, which are relatively difficult to study because of a) the vastness and threedimensional structure of the marine environment, making it difficult to sample; b) the difficulty of co-culturing marine algae and viruses in the laboratory; and c) the limited population size of experimental populations, where virus infection usually leads to a population crash. Second, in epidemiological models we can explore how aspects of laboratory rearing conditions themselves, such as batch cycle length or dilution rates for 
culture transfers, affect host-virus interactions. This in turn allows future studies to improve the design and the interpretation of laboratory experiments.

Here, we explore the impacts on the evolution of host resistance of two necessary simplifications of natural environments in laboratory experiments: population dynamics introduced by culturing method, and the lack of immigrants (re-introduction of virus) into host populations. To tackle these questions, we modelled the epidemiological dynamics of this system using a modified SIR (Susceptible-Infected-Resistant) model with different batch cycle lengths or dilution rates and under a range of costs of resistance. We test which magnitude of resistance costs can allow the coexistence of susceptible individuals within a mainly resistant population, and the sensitivity of model outputs to reseeding host populations with fresh viral particles. In particular, we answer the following questions: 1) How do rearing conditions such as growth cycles dominated by exponential vs stationary phase (corresponding to bloom vs non-bloom conditions in natural populations) and dilution rate (corresponding to strong or weak mixing of the water body) and virus re-seeding affect population dynamics of a host-virus system? 2) How does the cost of resistance affect the host-virus system in different rearing conditions? 3) Can we explain the discrepancies in the prevalence of host resistance between natural and laboratory systems?

\section{The model}

Simulations with one host population and one host-specific virus population

We consider an $O$. tauri/OtV population consisting of susceptible (S), infected (I) and resistant (R) host cells and viruses (V) (Fig. 1). Host cell population size is determined by a birth rate $b$ and a death rate $d$; resistant (R) cells experience a cost of resistance which reduces the birth rate by a constant rate $c$. All populations experience regulated growth determined by a carrying capacity $k$. We assume that the starting population already consists of susceptible and resistant cells, with susceptible cells present at a proportion of 100000:1, which is at the minimum of resistant cells as measured previously (Yau et al. 2016; 2018). Infectious $\mathrm{OtV}$ viruses are also present in the starting population at a proportion of 100 times less than the host population, as determined empirically in the laboratory (Heath SE., unpublished data). This model assumes the population is well mixed and that host $O$. tauri cells and OtVs are distributed homogenously and come into contact at random. The rate at which an encounter between a host cell and an OtV results in a successful infection is therefore proportional to the densities of the hosts and viruses and the infection rate $(\beta)$. 
149 Successful infection of susceptible cells usually results in cell death via lysis $(\lambda)$ with a burst

150

151

152

153

154

155

156

157

158

159

160

161

162

163

164

165

166

167

168

169

170

171

172

173

$$
\frac{d S}{d t}=b S\left(1-\frac{N}{k}\right)-d S-\beta S V
$$

$$
\frac{d I}{d t}=\beta \mathrm{SV}-\lambda \mathrm{I}-\alpha \mathrm{I}
$$

$$
\frac{d R}{d t}=b \mathrm{R}\left(1-\frac{N}{k}\right)(1-c)-d \mathrm{R}+\alpha \mathrm{I}
$$

$$
\frac{d V}{d t}=\lambda \delta \mathrm{I}-\beta \mathrm{SV}-\beta \mathrm{RV}-\gamma \mathrm{V}
$$

175 where total cell population size $\mathrm{N}=\mathrm{S}+\mathrm{I}+\mathrm{R}$. 
The model simulates a semi-continuous batch culture system where after a number of set integration steps (i.e. batch cycle length=BC) the population was diluted. In the remainder of this study, we will refer to the number of integration steps that elapse between dilutions as 'transfer rates'. The transfer rates varied between 2 days and 12 days. In addition, we used different quantities of the population for each transfer (i.e. different dilutions) that varied between 0.1 (10\% transferred from one batch cycle to the next) and 0.9 (90\% transferred, respectively). This allowed to test for the effect of changes to fundamental features of rearing conditions (how often cultures are transferred, and what proportion of the population is transferred) of the host-virus system. The model was then run for a length of 160 model days (integration steps) which led to a different number of maximum batch cycles between 16 (for long batch cycle lengths) and 80 (for short batch cycle lengths). The growth rate we used in the model was 1 which means that the maximum number of generations that a population has gone through was 160 when the model population did not reach stationary phase. Any longer runs would not be comparable to most published laboratory or natural observations in this system. Also, since evolutionary processes readily occur in laboratory experiments on this timescale (Koskella and Brockhurst 2014; Collins 2011), longer timescales were not explored here.

After investigating how rearing conditions affected the dynamics of host resistance assuming that resistance had no cost, we tested how a range of costs of resistance (between 0 and 1, with 0 referring to no cost, and 1 referring to death due to evolution of resistance) affected the modelled populations and the contributions of resistant (R) vs susceptible (S) host cells.

Extended simulations with the addition of new virus particles at an increasing rate:

Due to continuous mixing of the water column under natural conditions and strong host specificity, a resistant host cell can rapidly encounter new virus genotypes that may be infective, despite its resistance to previously encountered virus genotypes. We therefore extended our model such that at specific intervals we added a new virus population that was able to infect resistant host cells, and as such turns them into susceptible cells again (see supplementary material S1 for differential equations). The extended model contained a new susceptible host population (S2), a new infected population (I2) and a new virus population (V2) (Fig. S2 for extended model). We increased the rate of re-seeding the model population with new viruses as follows: The model was set up such that after either 1, 2, 4, or 8 batch cycles, the resistant host cells came into contact with a new virus (V2) to which they were susceptible. This turned them into susceptible cells (S2) that in turn could then become 
infected (I2). In the extended model we also tested how a range of costs of resistance (between 0 and 1) affected the modelled populations and the contributions of resistant vs. susceptible cells to the whole host population.

We performed all model simulations and approximations of ordinary equations using lsoda (Euler forward solution) in the deSolve package in $\mathrm{R}$ (version 3.3.1). Parameter values for the model are defined in Table 1 . The procedure of how data were extracted from the simulations and brought together to be shown in the results, is described in detail in the supplementary material S1.

\section{Results}

\section{Effect of transfer and dilution rate:}

In the first round of simulations assuming no cost of resistance, we tested how the transfer and dilution rates affected the host population and determined the conditions where the growth of host populations would remain in exponential phase or reach stationary phase in our simulation. We found that with higher dilution rates (fewer cells transferred), the host populations remained largely in exponential growth, which is in line with expectations from laboratory experiments. This was, however, only the case for fast transfer rates. Specifically, the longer the simulated batch cycle length, the more likely it was that the host populations reached stationary phase (Fig 2.), as expected. Based on these first explorations, we decided to run the subsequent simulations to test the effect of cost of resistance on the composition of host populations in all dilution scenarios and with batch cycle lengths of 4 and 10 days. We used all dilution scenarios to test how the loss of individuals during batch cycle transfers affected the model outcome. The different batch cycle lengths allowed the population to either remain in exponential growth (BC4) for the majority of the simulation, or to reach stationary phase (BC10) early in the simulation, respectively.

Effect of cost of resistance on several modelled populations:

Simulations with one host population and one host-specific virus population

We modelled how different costs of resistance affected the contribution of susceptible (S) and resistant (R) individuals to the host population. In the beginning of simulations (i.e. within one batch cycle) with fast transfer rates (BC 4, Fig. 3a) susceptible individuals were present in host populations. However, susceptible hosts were rapidly lost due to conversion to resistant individuals (Fig 3b-d) and did not change with different costs for resistance. Also, in the rest of the simulations (up to 160 integration steps) we found that including different costs 
of resistance did not affect the presence of susceptible individuals in the population. Instead, the cost of resistance affected the interaction of the stability of the resistant population and the culturing conditions. Specifically, increasing dilution rates (Figure 3, going from top to bottom in each panel) and increasing the cost of resistance (Figure 3, going from left to right in each panel) led to more experimental populations that collapsed (had a population size below 1000 cells). In addition, the longer the simulations were run (Figure 3, going from left to right panel), the more occasions there were where population collapse happened.

\section{Extended simulations with addition of new virus at an increasing rate:}

In the extended "virus re-seeding" version of the model, adding new viruses that were able to infect resistant host cells resulted in instances (i.e. simulations) where susceptible cells were present. This in itself is not surprising, but we also found that in the extended model the host populations were less stable. Populations became less stable with i) increasing the rate of re-seeding with viruses (Fig. 4 going from top to bottom), ii) increasing costs of resistance (Fig. 4 going from left to right in each panel), increasing the dilution and transfer rate (Fig. 4 going from top to bottom in each panel). In addition, in approximately $10 \%$ of the modelled cases, we found that susceptible cells re-grew in the host populations. That being said, the populations went extinct shortly after susceptible cells regrew. Due to the rapid infection of susceptible cells by the new viruses present in the population, there was not enough time for conversion to resistant cells to stabilize the population (Supplementary Material 2).

\section{Discussion}

Mathematical studies of microbial host-virus populations in the oceans extend previously established models, for example by adding a viral component to typical NPZ (nutrient-phytoplankton-zooplankton) food web models (e.g. Weitz et al. 2015) or by manipulating parameters in the Kill the Winner model (Thingstad 2000; Knowles et al. 2016). By using a SIR model, we were able to include the infected proportion of the host population, which is important in showing that $O$. tauri cells have two possible fates following virus infection (lysis or resistance). This allowed us to investigate the fates of host cells under different scenarios: a range of culturing conditions, costs of resistance and different introduction rates of new viruses into the systems.

Our modelling experiments show that culturing conditions can drastically affect experimental outcomes of host-virus dynamics with respect to populations' resilience to crashes and existence of resistant or susceptible hosts. Specifically, we showed that reaching 
stationary phase vs staying in exponential phase due to different transfer and dilution rates drastically altered the stability of the modelled populations. SIR models such as this one are thus a useful tool for the design of laboratory host-virus experiments that use batch cultures.

A general explanation for persistence of susceptible hosts within a population is that resistance to viruses is costly, and trades off with growth or reproduction (Antonovics et al. 1994; Sheldon and Verhulst 1996). When viruses are present in the environment, resistance is advantageous and selected for; however, in the absence of viruses, susceptible cells have a fitness advantage if resistance is costly, and should therefore rise in frequency (Lenski, R.E 1988). Previous work has shown costs of resistance to OtV to be very low or undetectable in $O$. tauri in the laboratory under a range of benign or ameliorated conditions (Thomas et al. 2011; Heath et al. 2017). However, since costs of resistance are context-dependent (Meaden, Paszkiewicz, and Koskella 2015; Koskella et al. 2012; Vale et al. 2015; Westra et al. 2015; Labbé, Vale, and Little 2010; Nystrand and Dowling 2020), it is possible that in the ocean, costs exist that have not or cannot be detected in the laboratory. Since we cannot rule out that resistance is in fact costly in natural populations of $O$. tauri, we investigated how varying the costs of resistance affected host populations. In our model, a cost of resistance higher than 0.15 always led to an extinction of the resistant population, which is consistent with laboratory studies failing to detect a cost that is in fact present in natural settings. One explanation for the absence of susceptible cells, when costs of resistance was higher than 0.15 , is the rapid infection of any susceptible cells within the starting population. In this case, the only way for the population to avoid extinction at the start of the simulation was indeed to become resistant as quickly as possible. If the cost of being resistant increased, the populations then shortly after went extinct. The transfer and dilution rates in the batch culture system modulated (slowed or speeded) this process, highlighting the context dependence of how costs of resistance affect host population dynamics. Thus, under laboratory conditions, it is not possible to maintain susceptible cells in the system when the evolution of resistance is irreversible. This is due to the uni-directional nature of resistance mutations in this system irrespective or the cost of resistance (Yau et al. 2016).

Another non-exclusive explanation for different results in field observations and modelling is that the dynamics of natural microbe-virus systems are strongly influenced by strain diversity (Agrawal and Lively 2002; Neiman and Fields 2016; Frank 1993), such that some aspects of the natural system cannot be modelled without incorporating diversity. If this is the case, models using single strains of hosts and viruses cannot capture key dynamics that drive the evolution of host resistance, and the maintenance of susceptible hosts, in wild 
312 populations. In particular, periodic exposure to new, infective virus strains is important for 313 maintaining susceptible host populations in our simulations. This is in line with current 314 knowledge about the diversity of Ostreococcus viruses. For example, Clerissi et al. isolated $31540 \mathrm{OtVs}$ that were strain specific, all but two of which came from Mediterranean lagoons 316 (Clerissi, Desdevises, and Grimsley 2012). When re-seeding the modelling experiment with 317 new viruses we then detected instances where susceptible cells were re-detected in the host 318 populations after resistance evolved. However, the frequency of re-detection and relative contribution of susceptible individuals was very low in our model, which is in contrast with the high prevalence of susceptible hosts in natural populations. As before, the frequency of re-detection and relative contribution was context-dependant because of the strong influence of culturing conditions.

Unsurprisingly, we point out that extrapolating from model simulations and laboratory studies to natural environments and interpretations needs to be done with caution. Our current model is not exhaustive and there are more variations that could play a role in how the model populations behave (here we highlight two): 1) different lengths of simulations/experiments and 2) varying numbers of host-virus pairs.

With respect to the length of simulations, we modelled the populations to up to 160 days of experiment. Considering that phytoplankton have fast generation times (in this case up to 1 generation/integration step) and viruses even faster generation times, 160 days of modelled experiment allows reasonable scope for mutations to occur (Elena and Lenski 2003). Fluctuating selection (i.e. the reciprocal evolution of hosts and viruses in response to each other in a negative frequency dependence), causes different rather than increased resistance and infectivity ranges, respectively, with average fitness remaining constant (Buckling and Rainey 2002; Avrani, Schwartz, and Lindell 2012). If fluctuating selection occurs with $O$. tauri and OtVs, this could explain how we do not see decreased fitness in resistant populations. Alternatively, the cost of resistance could be manifested as a limit in the number of virus strains against which a host can be resistant, or as increased susceptibility to other viral strains (Avrani et al. 2011; Marston et al. 2012). However, recent laboratory studies testing resistance specificity in OtV-resistant lines showed an increase in their range of resistance to viruses, probably because resistance to one strain is expected to give rise to resistance to genetically closely related viruses (Yau et al. 2018).

Regarding the point raised with respect to expanding the model: we are only considering a single host - single virus "pair" that is vastly underestimating natural diversity.

345 An individual based modelling approach (Beckmann, Schaum, and Hense 2019; Hinners, 
346 Hense, and Kremp 2019) could be better suited to tackling the question of how diversity

347 (either via starting diversity or evolved diversity via mutations) in both host and virus

348 populations more comprehensively affects host-virus dynamics.

349

350

\section{Conclusion:}

351

We have described an epidemiological SIR model to investigate $O$. tauri/OtV5

352 infection dynamics, with a focus on the effect of culturing conditions on the host-virus

353 dynamics. We demonstrate the application of traditional epidemiological models as a useful

354 approach to study marine algae/virus systems and build on data collected from the laboratory 355 and the field. 
359

360

361

362

363

364

365

366

367

368

369

370

371

372

373

374

375

376

377

378

379

380

381

382

383

384

385

386

387

388

389

390

391

392

393

394

395

396

397

\section{Acknowledgements}

S.E. Heath was supported by a BBSRC EASTBIO Doctoral Training Programme grant, P. Vale is supported by a Chancellor's fellowship (University of Edinburgh) and a Branco Weiss fellowship from Society in Science (https://brancoweissfellowship.org/). S. Collins was supported by a Royal Society University Research Fellowship during part of this project...Luisa Listmann and Elisa Schaum are supported by a start-up grant to ES by Universität Hamburg.

\section{References}

Agrawal, Aneil, and Curtis M Lively. 2002. "Infection Genetics: Gene-for-Gene versus Matching-Alleles Models and All Points in Between."

Antonovics, Janis, Peter H Thrall, Janis Antonovics, and Peter H Thrall. 1994. "The Cost of Resistance and the Maintenance of Genetic Polymorphism in Host-Pathogen Systems" 257 (1349): 105-10.

Avrani, Sarit, Daniel A. Schwartz, and Debbie Lindell. 2012. "Virus-Host Swinging Party in the Oceans." Mobile Genetic Elements 2 (2): 88-95. https://doi.org/10.4161/mge.20031.

Avrani, Sarit, Omri Wurtzel, Itai Sharon, Rotem Sorek, and Debbie Lindell. 2011. "Genomic Island Variability Facilitates Prochlorococcusvirus Coexistence.” Nature 474 (7353): 604-8. https://doi.org/10.1038/nature10172.

Beckmann, Aike, C. Elisa Schaum, and Inga Hense. 2019. "Phytoplankton Adaptation in Ecosystem Models." Journal of Theoretical Biology 468 (May): 60-71. https://doi.org/10.1016/j.jtbi.2019.01.041.

Brussaard, Corina P.D. 2004. "Viral Control of Phytoplankton Populations - A Review." In Journal of Eukaryotic Microbiology, 51:125-38. John Wiley \& Sons, Ltd. https://doi.org/10.1111/j.15507408.2004.tb00537.x.

Buckling, A., and P. B.. Rainey. 2002. "Antagonistic Coevolution between a Bacterium and a Bacteriophage." Proceedings of the Royal Society of London. Series B: Biological Sciences 269 (1494): 931-36. https://doi.org/10.1098/rspb.2001.1945.

Clerissi, Camille, Yves Desdevises, and Nigel Grimsley. 2012. "Prasinoviruses of the Marine Green Alga Ostreococcus Tauri Are Mainly Species Specific.” Journal of Virology 86 (8): 4611-19. https://doi.org/10.1128/JVI.07221-11.

Clerissi, Camille, Nigel Grimsley, Hiroyuki Ogata, Pascal Hingamp, Julie Poulain, and Yves Desdevisesa. 2014. "Unveiling of the Diversity of Prasinoviruses (Phycodnaviridae) in Marine Samples by Using HighThroughput Sequencing Analyses of PCR-Amplified DNA Polymerase and Major Capsid Protein Genes." Applied and Environmental Microbiology 80 (10): 3150-60. https://doi.org/10.1128/AEM.00123-14.

Clerissi, Camille, Nigel Grimsley, Lucie Subirana, Eric Maria, Louise Oriol, Hiroyuki Ogata, Hervé Moreau, and Yves Desdevises. 2014. "Prasinovirus Distribution in the Northwest Mediterranean Sea Is Affected by the Environment and Particularly by Phosphate Availability." Virology 466-467 (October): 146-57. https://doi.org/10.1016/j.virol.2014.07.016.

Collins, Sinéad. 2011. "Many Possible Worlds: Expanding the Ecological Scenarios in Experimental Evolution.” Evolutionary Biology 38 (1): 3-14. https://doi.org/10.1007/s11692-010-9106-3.

Courties, Claude, André Vaquer, Marc Troussellier, Jacques Lautier, Marie J. Chrétiennot-Dinet, Jacques 

(6487): 255-255. https://doi.org/10.1038/370255a0.

400

401

402

403

404

405

406

407

408

409

410

411

412

413

414

415

416

417

418

419

420

421

422

423

424

425

426

427

428

429

430

431

432

433

434

Derelle, E., C. Ferraz, S. Rombauts, P. Rouze, A. Z. Worden, S. Robbens, F. Partensky, et al. 2006. "Genome Analysis of the Smallest Free-Living Eukaryote Ostreococcus Tauri Unveils Many Unique Features." Proceedings of the National Academy of Sciences 103 (31): 11647-52. https://doi.org/10.1073/pnas.0604795103.

Derelle, Evelyne, Conchita Ferraz, Marie Line Escande, Sophie Eychenié, Richard Cooke, Gwenaël Piganeau, Yves Desdevises, Laure Bellec, Harvé Moreau, and Nigel Grimsley. 2008. "Life-Cycle and Genome of OtV5, a Large DNA Virus of the Pelagic Marine Unicellular Green Alga Ostreococcus Tauri." PLoS ONE 3 (5). https://doi.org/10.1371/journal.pone.0002250.

Elena, Santiago F., and Richard E. Lenski. 2003. "Evolution Experiments with Microorganisms: The Dynamics and Genetic Bases of Adaptation." Nature Reviews Genetics. https://doi.org/10.1038/nrg1088.

Frank, S. A. 1993. "Specificity versus Detectable Polymorphism in Host-Parasite Genetics." Proceedings of the Royal Society B: Biological Sciences 254 (1341): 191-97. https://doi.org/10.1098/rspb.1993.0145.

Gandon, S., and P. F. Vale. 2014. “The Evolution of Resistance against Good and Bad Infections.” Journal of Evolutionary Biology 27 (2): 303-12. https://doi.org/10.1111/jeb.12291.

Grassly, Nicholas C., and Christophe Fraser. 2008. "Mathematical Models of Infectious Disease Transmission." Nature Reviews Microbiology 6 (6): 477-87. https://doi.org/10.1038/nrmicro1845.

Heath, Sarah E., Kirsten Knox, Pedro F. Vale, and Sinead Collins. 2017. "Virus Resistance Is Not Costly in a Marine Alga Evolving under Multiple Environmental Stressors." Viruses 9 (3). https://doi.org/10.3390/v9030039.

Heesterbeek, H., R. M. Anderson, V. Andreasen, S. Bansal, D. De Angelis, C. Dye, K. T. D. Eames, et al. 2015. "Modeling Infectious Disease Dynamics in the Complex Landscape of Global Health." Science 347 (6227): aaa4339-aaa4339. https://doi.org/10.1126/science.aaa4339.

Hinners, Jana, Inga Hense, and Anke Kremp. 2019. "Modelling Phytoplankton Adaptation to Global Warming Based on Resurrection Experiments." Ecological Modelling 400 (March): 27-33. https://doi.org/10.1016/j.ecolmodel.2019.03.006.

Knowles, B., C. B. Silveira, B. A. Bailey, K. Barott, V. A. Cantu, A. G. Cobian-Guëmes, F. H. Coutinho, et al. 2016. "Lytic to Temperate Switching of Viral Communities." Nature 531 (7595): 466-70. https://doi.org/10.1038/nature17193.

Koskella, Britt, and Michael A. Brockhurst. 2014. "Bacteria-Phage Coevolution as a Driver of Ecological and Evolutionary Processes in Microbial Communities." FEMS Microbiology Reviews 38 (5): 916-31. https://doi.org/10.1111/1574-6976.12072.

Koskella, Britt, Derek M. Lin, Angus Buckling, and John N. Thompson. 2012. "The Costs of Evolving Resistance in Heterogeneous Parasite Environments." Proceedings of the Royal Society B: Biological Sciences 279 (1735): 1896-1903. https://doi.org/10.1098/rspb.2011.2259.

Labbé, Pierrick, Pedro F. Vale, and Tom J. Little. 2010. "Successfully Resisting a Pathogen Is Rarely Costly in Daphnia Magna." BMC Evolutionary Biology 10 (1): 1-12. https://doi.org/10.1186/1471-2148-10-355.

Lenski, Richard E. 1988. "Dynamics of Interactions between Bacteria and Virulent Bacteriophage." In , 1-44. Springer, Boston, MA. https://doi.org/10.1007/978-1-4684-5409-3_1. 
Marston, Marcia F., Francis J. Pierciey, Alicia Shepard, Gary Gearin, Ji Qi, Chandri Yandava, Stephan C. Schuster, Matthew R. Henn, and Jennifer B.H. Martiny. 2012. "Rapid Diversification of Coevolving Marine Synechococcus and a Virus." Proceedings of the National Academy of Sciences of the United States of America 109 (12): 4544-49. https://doi.org/10.1073/pnas.1120310109.

Meaden, Sean, Konrad Paszkiewicz, and Britt Koskella. 2015. "The Cost of Phage Resistance in a Plant Pathogenic Bacterium Is Context-dependent.” Evolution 69 (5): 1321-28. https://doi.org/10.1111/evo.12652.

Middelboe, M. 2000. "Bacterial Growth Rate and Marine Virus-Host Dynamics.” Microbial Ecology 40 (2): 114-24. https://doi.org/10.1007/s002480000050.

Neiman, M., and P. Fields. 2016. “Antagonistic Interspecific Coevolution.” In Encyclopedia of Evolutionary Biology, 93-100. Elsevier Inc. https://doi.org/10.1016/B978-0-12-800049-6.00196-7.

Nystrand, M., and D. K. Dowling. 2020. "Effects of Immune Challenge on Expression of Life-History and Immune Trait Expression in Sexually Reproducing Metazoans - A Meta-Analysis.” BMC Biology 18 (1): 1-17. https://doi.org/10.1186/s12915-020-00856-7.

Schroeder, Declan C., Joanne Oke, Matthew Hall, Gillian Malin, and William H. Wilson. 2003. "Virus Succession Observed during an Emiliania Huxleyi Bloom.” Applied and Environmental Microbiology 69 (5): 2484-90. https://doi.org/10.1128/AEM.69.5.2484-2490.2003.

Sheldon, Ben C., and Simon Verhulst. 1996. "Ecological Immunology: Costly Parasite Defences and TradeOffs in Evolutionary Ecology." Trends in Ecology and Evolution. Elsevier Current Trends. https://doi.org/10.1016/0169-5347(96)10039-2.

Short, Steven M. 2012. “The Ecology of Viruses That Infect Eukaryotic Algae.” Environmental Microbiology 14 (9): 2253-71. https://doi.org/10.1111/j.1462-2920.2012.02706.x.

Šulčius, Sigitas, and Karin Holmfeldt. 2016. "Viruses of Microorganisms in the Baltic Sea: Current State of Research and Perspectives.” Marine Biology Research 12 (2): 115-24.

Suttle, Curtis A. 2005. "Viruses in the Sea." Nature. Nature Publishing Group. https://doi.org/10.1038/nature04160.

—. 2007. "Marine Viruses - Major Players in the Global Ecosystem.” Nature Reviews Microbiology 5 (10): 801-12. https://doi.org/10.1038/nrmicro1750.

Thingstad, T. F. 2000. "Elements of a Theory for the Mechanisms Controlling Abundance, Diversity, and Biogeochemical Role of Lytic Bacterial Viruses in Aquatic Systems.” Limnology and Oceanography 45 (6): 1320-28. https://doi.org/10.4319/lo.2000.45.6.1320.

Thomas, Rozenn, Nigel Grimsley, Marie line Escande, Lucie Subirana, Evelyne Derelle, and Hervé Moreau. 2011. "Acquisition and Maintenance of Resistance to Viruses in Eukaryotic Phytoplankton Populations." Environmental Microbiology 13 (6): 1412-20. https://doi.org/10.1111/j.1462-2920.2011.02441.x.

Vale, Pedro F., Guillaume Lafforgue, Francois Gatchitch, Rozenn Gardan, Sylvain Moineau, and Sylvain Gandon. 2015. "Costs of CRISPR-Cas-Mediated Resistance in Streptococcus Thermophilus.” Proceedings of the Royal Society B: Biological Sciences 282 (1812): 20151270. https://doi.org/10.1098/rspb.2015.1270.

Weitz, Joshua S., Charles A. Stock, Steven W. Wilhelm, Lydia Bourouiba, Maureen L. Coleman, Alison Buchan, Michael J. Follows, et al. 2015. “A Multitrophic Model to Quantify the Effects of Marine Viruses 
on Microbial Food Webs and Ecosystem Processes." ISME Journal 9 (6): 1352-64.

Weitz, Joshua S., and Steven W. Wilhelm. 2012. “Ocean Viruses and Their Effects on Microbial Communities and Biogeochemical Cycles." F1000 Biology Reports 4 (1): 17. https://doi.org/10.3410/B4-17.

Westra, Edze R., Stineke Van Houte, Sylvain Gandon, and Rachel Whitaker. 2019. "The Ecology and Evolution of Microbial CRISPR-Cas Adaptive Immune Systems." Philosophical Transactions of the Royal Society B: Biological Sciences. Royal Society Publishing. https://doi.org/10.1098/rstb.2019.0101.

Westra, Edze R., Stineke Van houte, Sam Oyesiku-Blakemore, Ben Makin, Jenny M. Broniewski, Alex Best, Joseph Bondy-Denomy, Alan Davidson, Mike Boots, and Angus Buckling. 2015. "Parasite Exposure Drives Selective Evolution of Constitutive versus Inducible Defense." Current Biology 25 (8): 1043-49. https://doi.org/10.1016/j.cub.2015.01.065.

Worden, Alexandra Z., Jessica K. Nolan, and B. Palenik. 2004. “Assessing the Dynamics and Ecology of Marine Picophytoplankton: The Importance of the Eukaryotic Component." Limnology and Oceanography 49 (1): 168-79. https://doi.org/10.4319/1o.2004.49.1.0168.

Yau, Sheree, Gaëtan Caravello, Nadège Fonvieille, Élodie Desgranges, Hervé Moreau, and Nigel Grimsley. 2018. "Rapidity of Genomic Adaptations to Prasinovirus Infection in a Marine Microalga." Viruses 10 (8): $1-11$. Viral Immunity Chromosome in the Marine Picoeukaryote, Ostreococcus Tauri." PLoS Pathogens 12 (10): 1-25. https://doi.org/10.1371/journal.ppat.1005965. 
504 Table1: Parameter input to SIR-model

$\begin{array}{llll}\text { Parameter } & \text { Description } & \text { Value } & \text { Reference } \\ \boldsymbol{B} & \text { Birth } & 1 \text { division per day } & \text { Heath \& Collins 2016 } \\ \boldsymbol{D} & \text { Death } & 0.05 \text { per cell per day } & \text { Estimate } \\ \boldsymbol{\beta} & \text { Infection rate } & 0.35 \text { per cell per day } & \text { Derelle } \text { et al. } 2008 \\ \boldsymbol{A} & \text { Lysis rate } & 0.99 \text { per cell per day } & \text { Estimated based on Derelle et al. } \\ & & 2008 \\ \boldsymbol{A} & \text { Conversion rate of I to R } & 0.01 \text { per cell per day } & \text { Yau } \text { et al. 2016 } \\ \boldsymbol{\Gamma} & \text { Virus decay rate } & 0.01 \text { per virus per day } & \text { This study } \\ \boldsymbol{\delta} & \text { Burst size } & 25 \text { per cell day } & \text { Derelle } \text { et al. } 2008 \\ \boldsymbol{C} & \text { Cost of resistance } & 0-1 & \text { This study } \\ \boldsymbol{K} & \text { Carrying capacity } & 1000000 & \text { This study } \\ \boldsymbol{B C} & \text { Batch cycle length } & 2-12 \text { days } & \text { This study } \\ \boldsymbol{D i l} & \text { Dilution rate } & 0.1-0.9 & \text { This study }\end{array}$

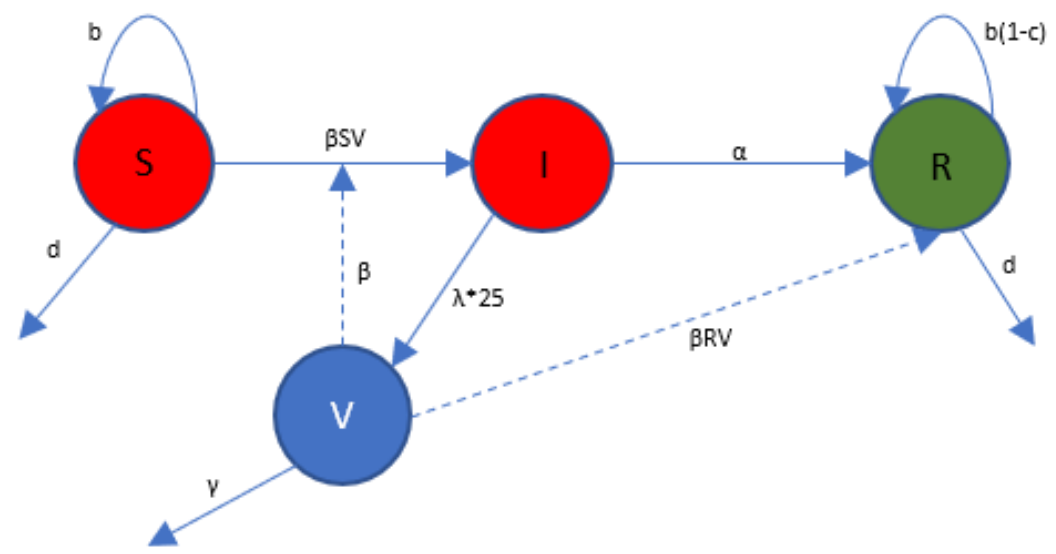

Figure 1. Flow diagram of the model. Circles represent different states of susceptible (S), infected (I) that are also counted into the susceptible population, and resistant (R) states of $O$. tauri cells and viruses (V). Dotted lines indicate effects of viral absorption whereas arrows indicate transitions between states. 


\section{Resistant individuals Susceptible individuals} BC2 BC4 BC6 BC8 BC10 $\mathrm{BC} 12$

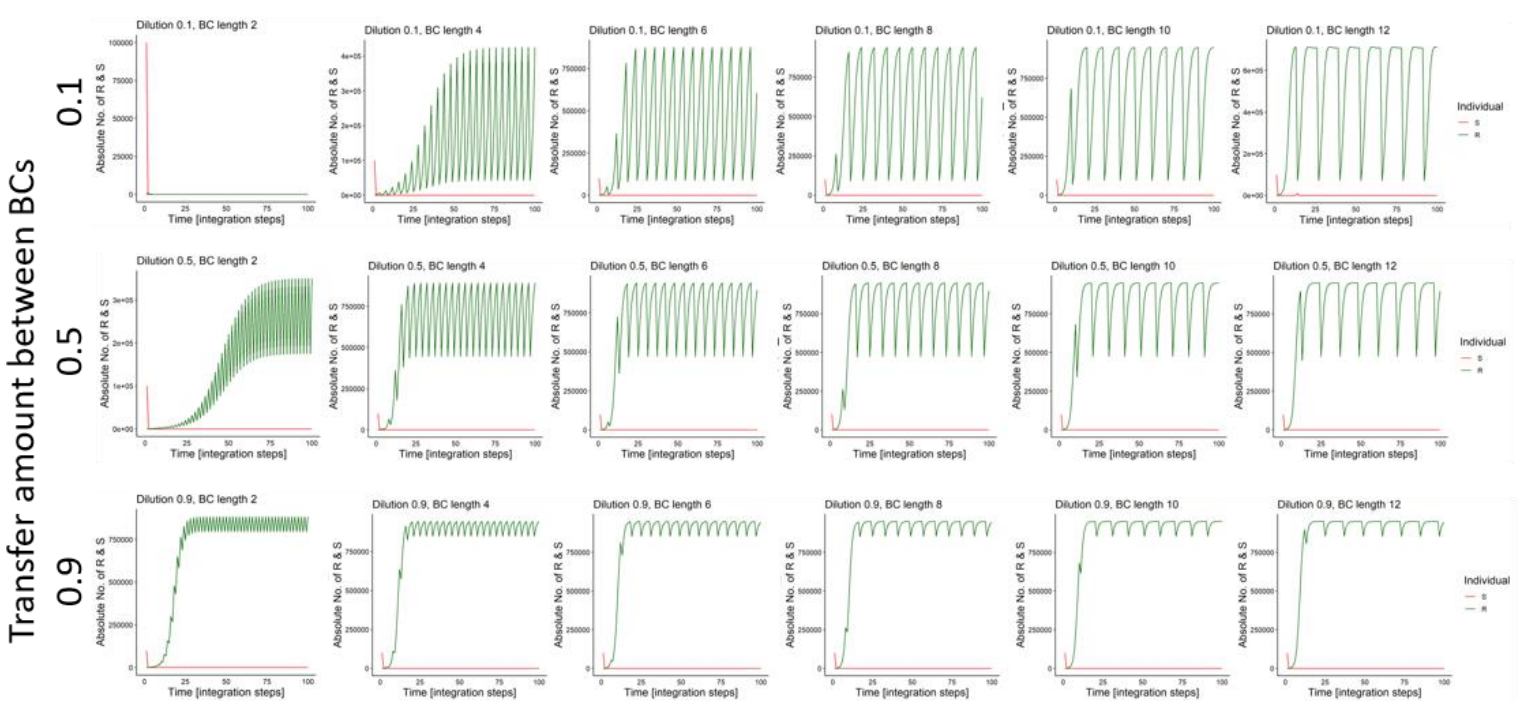

Figure 2. Here the effect of transfer amount ( 0.1 to 0.9 from one batch cycle to another, with 0.1 indicating a transfer of $10 \%$ and 0.9 indicating $90 \%$ of the model culture transferred, respectively) (top to bottom) and batch cycle lengths (BC) (left to right) are shown over the model simulations. The number of resistant cells are depicted in green whereas the number of susceptible individuals are depicted in red.
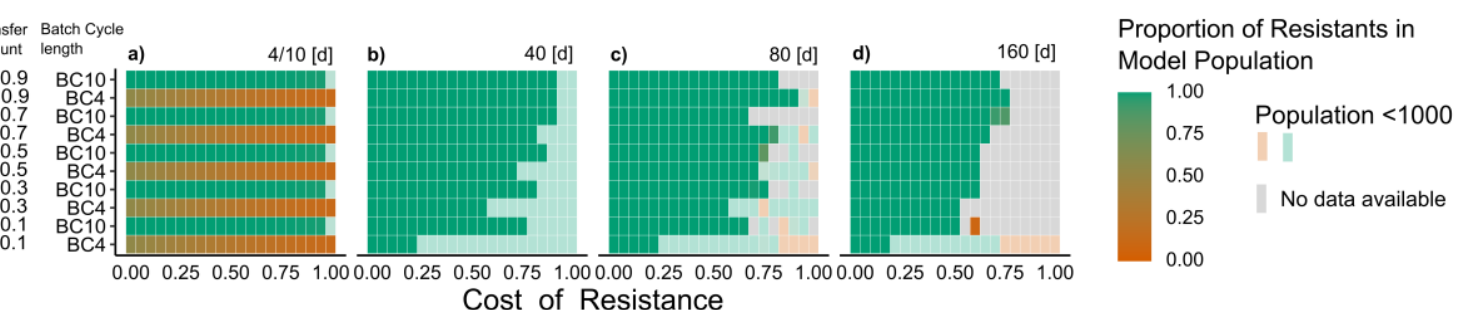

Figure 3. The relative proportion of resistant individuals in the experimental population (green) are shown at

522 the end of the first batch cycle (a) and after 40-160 (b-c) days of modelling run. We note that the number of

523 batch cycles (i.e. transfers) vary for the number of experiments between 4-16 batch cycles when the length is 10

524 days and between 10 to 80 batch cycles when the length is 4 days. On the $\mathrm{x}$-axis the different modelled costs of

525 resistance are depicted whereas on the y-axis the different culturing conditions are depicted. The colours depict

526 the proportion of resistant (green) vs. susceptible (red) cells in the model populations. When the model

527 population was lower than 1000 individuals the population was defined as "collapsed". 


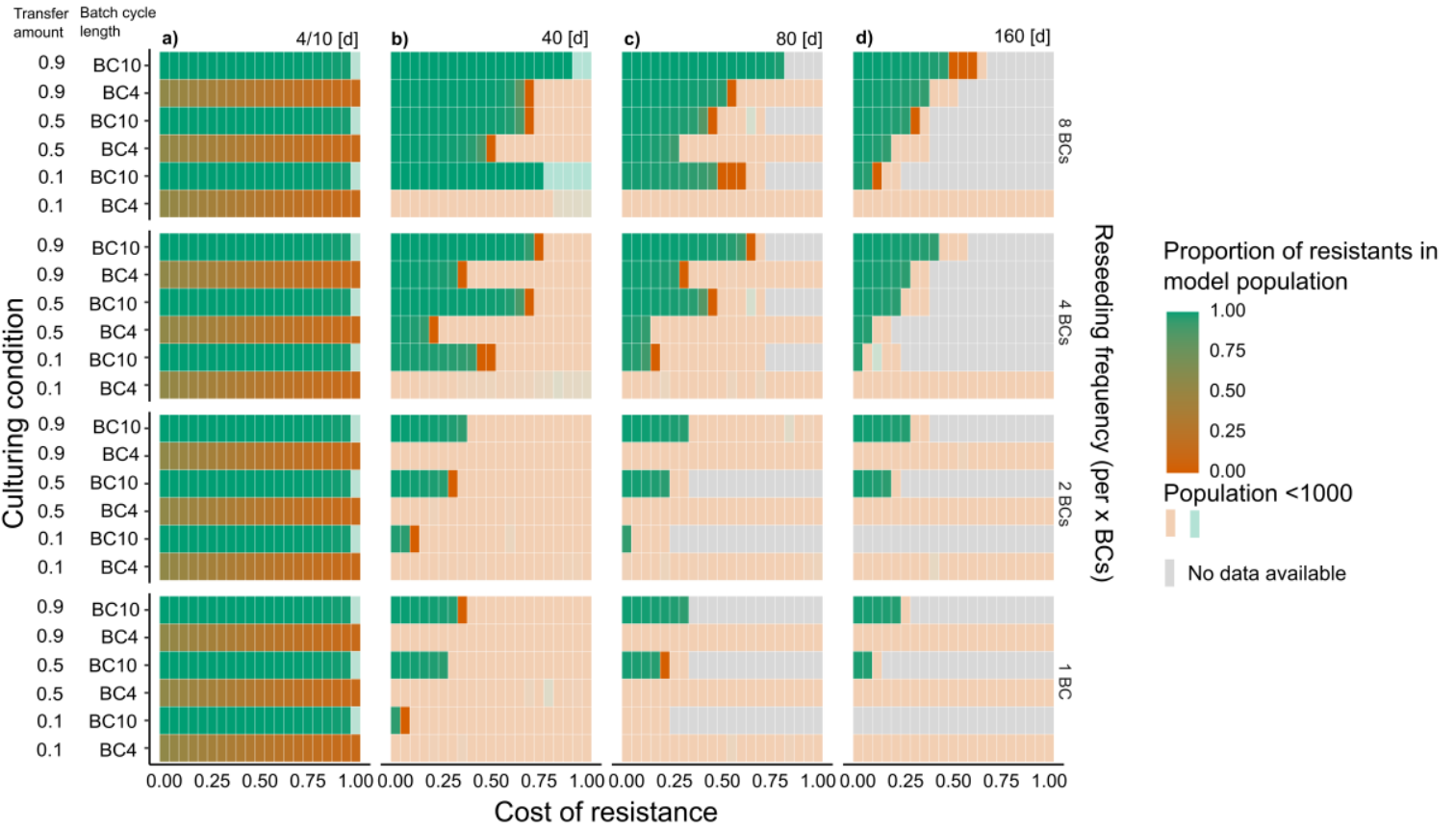

530 Figure 4. The relative proportion of resistant individuals in the experimental population (green) are shown at 531 the end of the first batch cycle (a) and after 40-160 days (c-d) of modelling run. We note that the number of 532 batch cycles (i.e. transfers) vary for the number of experiments between 4-16 batch cycles when the length is 10 533 days and between 10 to 80 batch cycles when the length is 4 days. On the $\mathrm{x}$-axis the different modelled costs of 534 resistance are depicted whereas on the y-axis the different culturing conditions are depicted. Going from the top 535 to bottom row the modelling results with increasing virus re-seeding frequency are shown. The colours depict 536 the proportion of resistant (green) vs susceptible (red) cells in the model populations. When the model population was lower than 1000 individuals the population was defined as "collapsed". 
Resistant individuals Susceptible individuals

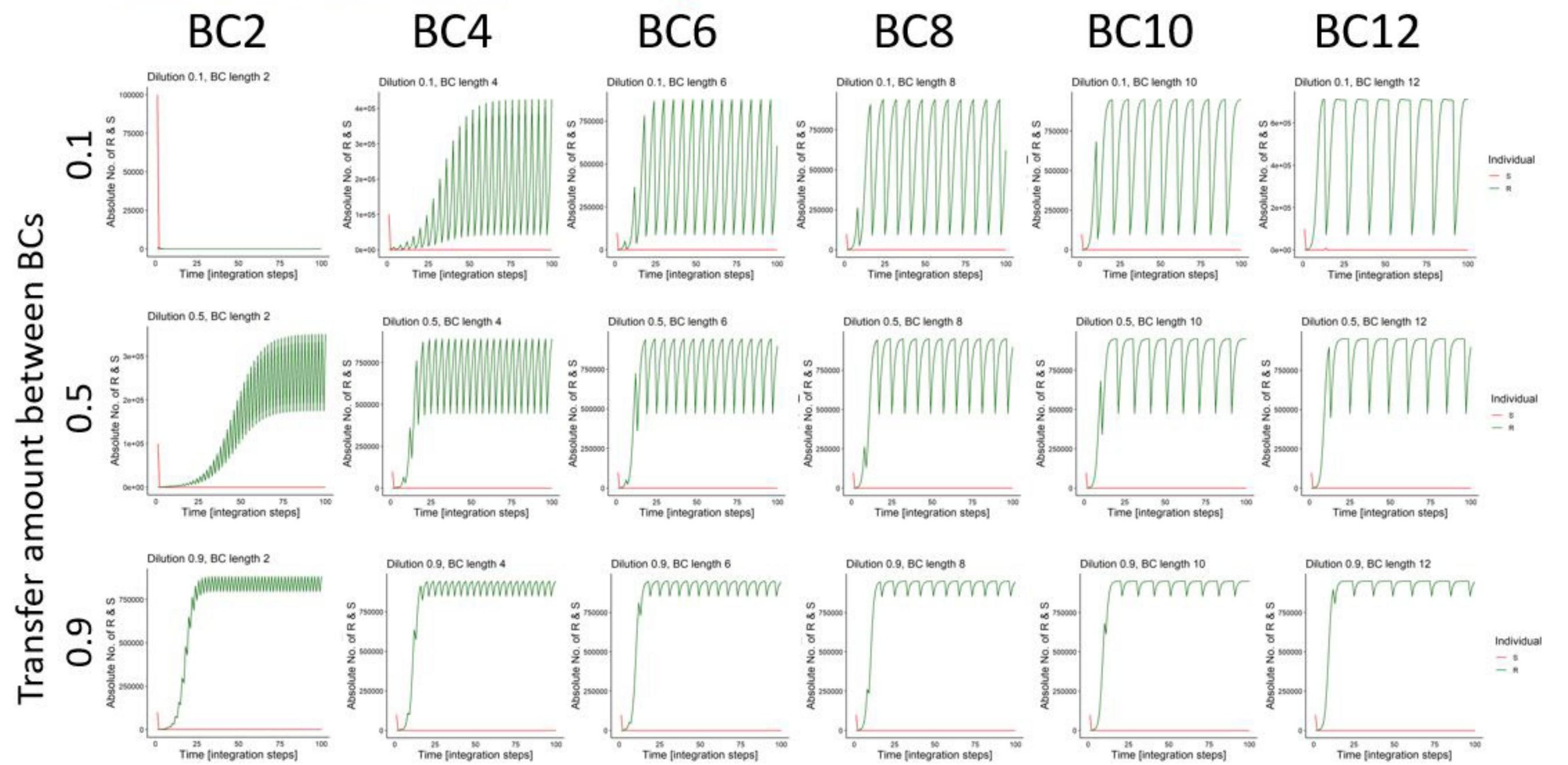


Transfer Batch Cycle

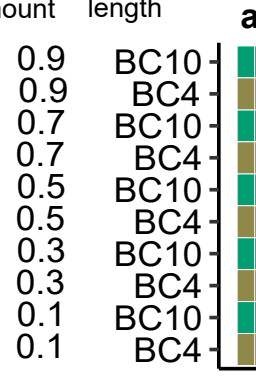

a)

4/10 [d]

b)

40 [d]

c)

$80[\mathrm{~d}]$

d)

$160[\mathrm{~d}]$
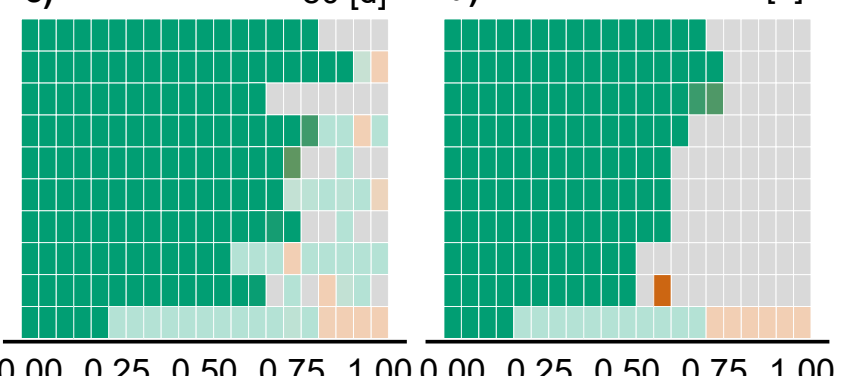

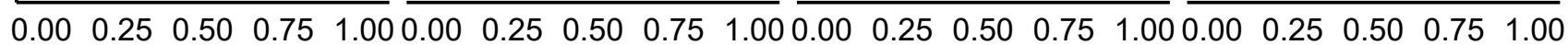
Cost_of_Resistance
Proportion of Resistants in Model Population

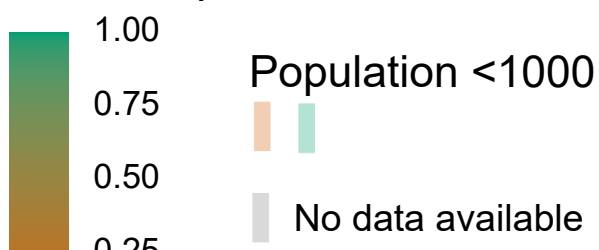


a)

$\begin{array}{rr}0.9 & \mathrm{BC} 10 \\ 0.9 & \mathrm{BC} 4 \\ 0.5 & \mathrm{BC} 10 \\ 0.5 & \mathrm{BC} 4 \\ 0.1 & \mathrm{BC} 10 \\ 0.1 & \mathrm{BC} 4\end{array}$

\begin{tabular}{|c|c|c|}
\hline & 0.9 & BC10 \\
\hline & 0.9 & BC4 \\
\hline 0 & 0.5 & $\mathrm{BC} 10$ \\
\hline & 0.5 & $\mathrm{BC}$ \\
\hline$\overline{0}$ & 0.1 & $\mathrm{BC} 10$ \\
\hline & 0.1 & $\mathrm{~B}$ \\
\hline
\end{tabular}
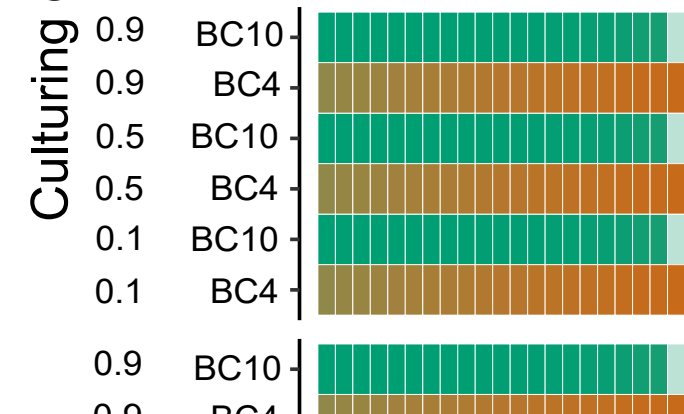

0.9 BC4

0.5 BC10

0.5 BC4

0.1 BC10

$0.1 \quad B C 4$
4/10 [d] b)
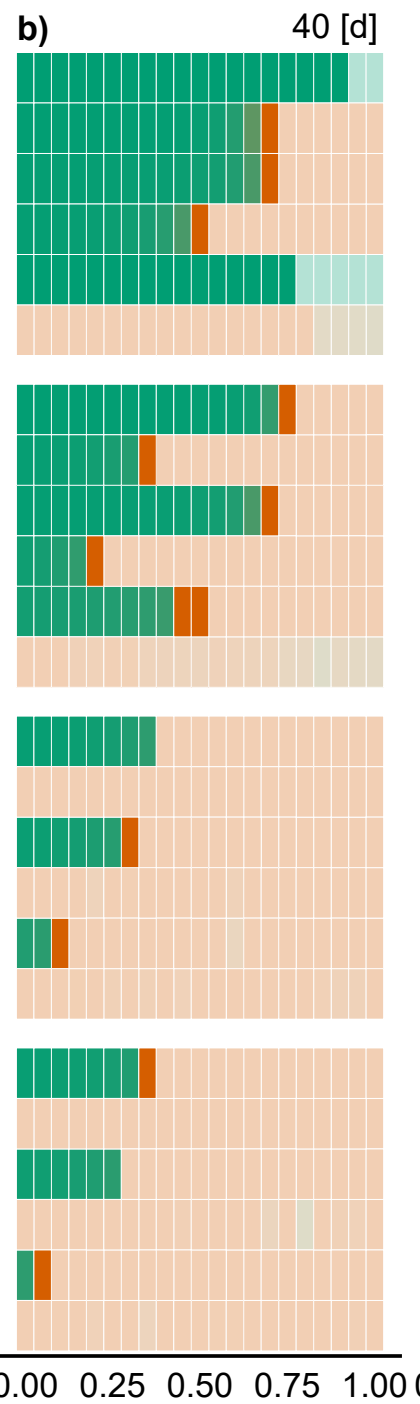

Cost of resistance $80[\mathrm{~d}]$

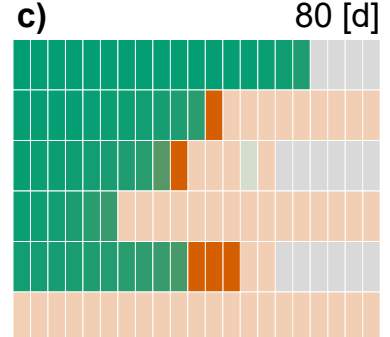

d)

$160[d]$
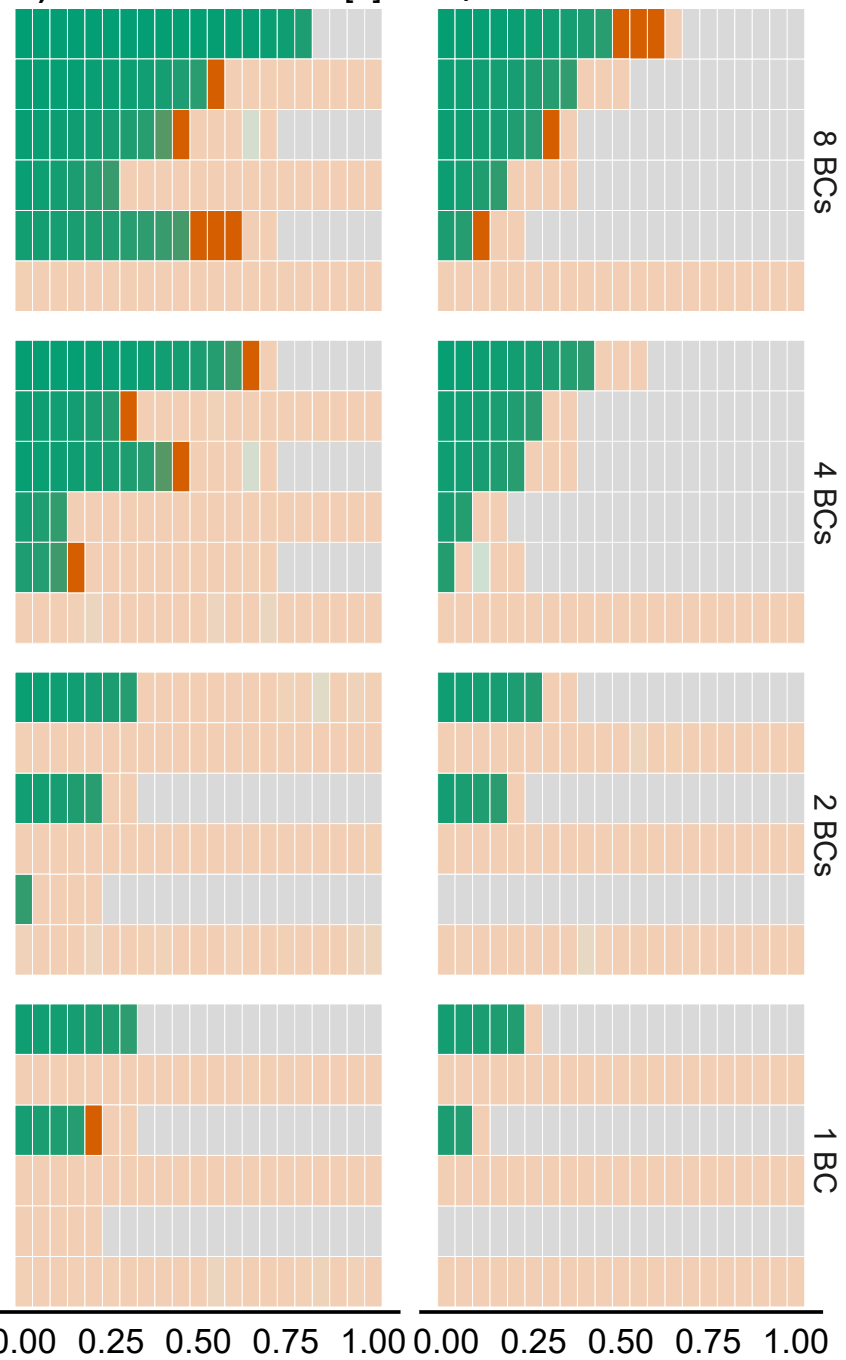

$\overrightarrow{\mathrm{D}}$
Proportion of resistants in model population

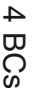

बै

$$
\begin{aligned}
& 1.00 \\
& 0.75 \\
& 0.50 \\
& 0.25 \\
& 0.00
\end{aligned}
$$

Population $<1000$<smiles>C1CCC1</smiles>

No data available 


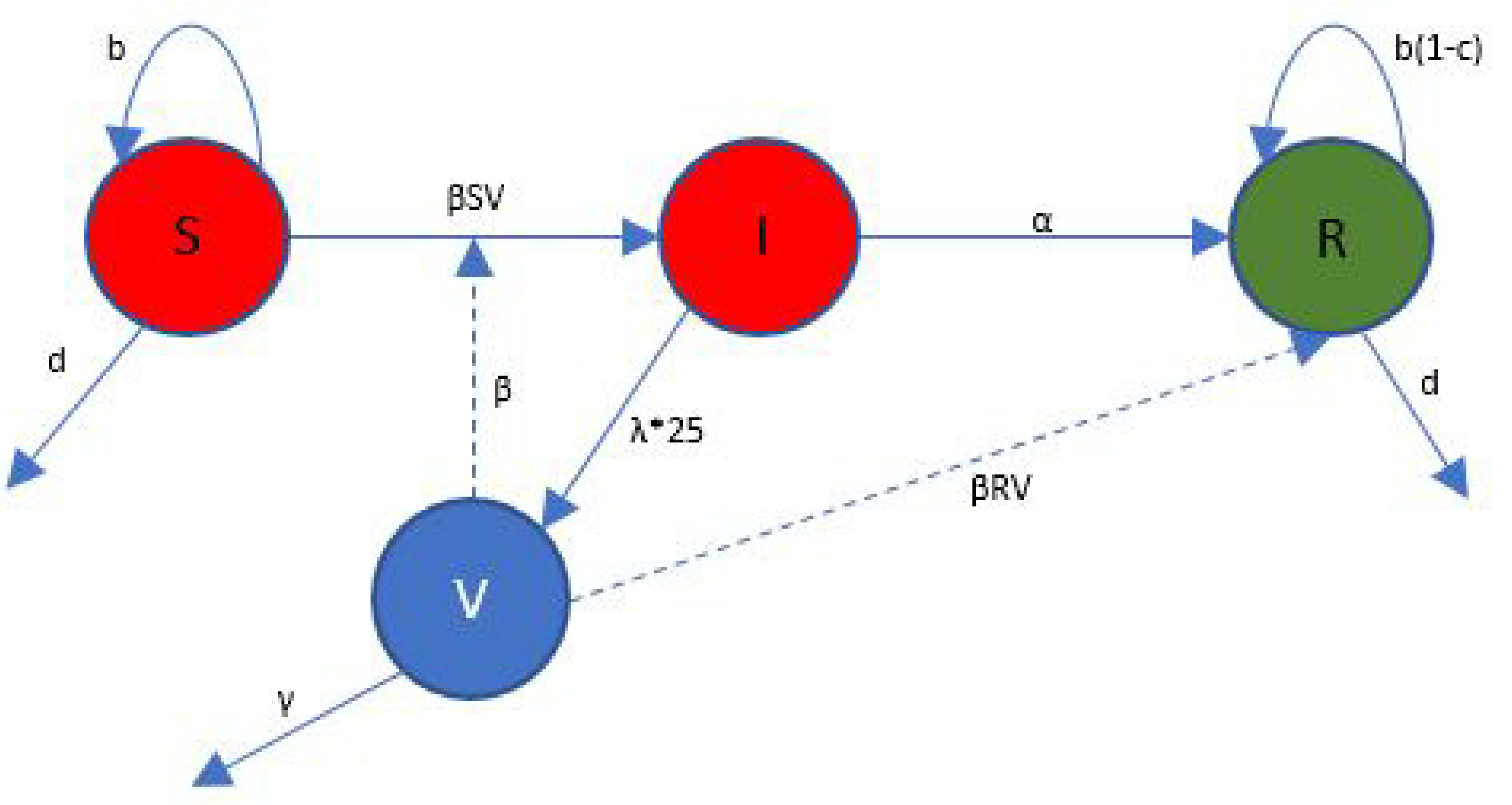

